\title{
SOME RESULTS ON RIGIDITY OF HOLOMORPHIC MAPPINGS
}

\author{
By Yoshinisa KuBota
}

1. In this paper we study rigidity properties of holomorphic mappings. Let $X$ and $Y$ be complex normed spaces. Let $D_{1}$ be a balanced domain in $X$ and $D_{2}$ be a bounded convex balanced domain in $Y$. We consider holomorphic mappings $f$ from $D_{1}$ into $D_{2}$. We prove two theorems. One of them is a generalization of the Schwarz lemma, which gives an upper bound for $\mu_{D_{2}}(f(x))$, $x \in D_{1}$. Here $\mu_{D_{2}}$ denotes the Minkowski functional of $D_{2}$. We also discuss the extremal mappings related to the Schwarz lemma. We deduce as a corollary the following fact: if $f: X \rightarrow Y$ is a holomorphic mapping which satisfies $\|f(x)\|$ $=\|x\|$ for all $x \in X$, then $f$ is linear. Another theorem gives a lower bound for $\mu_{D_{2}}(f(x)), x \in D_{1}$. Finally we are concerned with the limits of sequences of automorphisms of bounded domains. It is known that if $D$ is a bounded domain in $C^{n}$ and if a mapping $f: D \rightarrow D$ is a pointwise limit of a sequence of automorphisms of $D$, then $f$ is also an automorphism of $D$. However, in the case that $D$ is a bounded domain in a complex normed space $X$ the limit $f: D \rightarrow D$ need not be an automorphism of $D$. We give a simple counterexample. Using the above two theorems we show that the limit $f$ is one-to-one.

2. We summarize the main notation and terminology used in this paper. Let $X$ be a complex normed space and let $D$ be a domain in $X$. The Minkowski functional $\mu_{D}$ of $D$ is defined by

$$
\mu_{D}(x)=\inf \left\{t>0: t^{-1} x \in D\right\} \quad(x \in X) .
$$

We denote the open ball with center at $a$ and radius $r$ in $X$ by $B(a, r)$. Then we have that $\mu_{B(0, r)}(x)=r^{-1}\|x\|$.

Let $X$ and $Y$ be complex normed spaces and let $D$ be a domain in $X$. A mapping $f: D \rightarrow Y$ is said to be holomorphic in $D$ if, corresponding to every $a \in D$, there exist a power series $\sum_{k=0}^{\infty} P_{k}$ and a positive number $\rho$ such that $f$ is expressed by

$$
f(x)=\sum_{k=0}^{\infty} P_{k}(x-a) \quad(x \in B(a, \rho)) .
$$

Received May 7, 1993; revised October 29, 1993. 
Here $P_{k}$ is a continuous $k$-homogeneous polynomial from $X$ to $Y$, and the convergence is uniform on $B(a, r)$ for every $r$ with $0<r<\rho$. We use the notation

$$
\hat{d}^{k} f(a)=k ! P_{k} \quad(k=0,1,2, \cdots),
$$

and we call the power series

$$
\sum_{k=0}^{\infty} \frac{1}{k !} \hat{d}^{k} f(a)(x-a)
$$

the Taylor series of $f$ at $a$. We refer to [1], [2] and [6] for further details.

3. There are some generalizations of the Schwarz lemma in complex normed spaces (see, for example, [2], [3], [4] and[5]). We also give a generalization of the Schwarz lemma. We adapt Rudin's proof [8, Theorem 8.1.2] in which only the finite dimensional case is considered.

Let $X$ and $Y$ be complex normed spaces and let $D_{1}$ and $D_{2}$ be balanced domains in $X$ and $Y$, respectively. Suppose that $D_{1} \neq X$. Then there exists a point $x \in X$ with $\mu_{D_{1}}(x)>0$. For a holomorphic mapping $f: D_{1} \rightarrow D_{2}$ we define

and

$$
\hat{\lambda}_{f}=\inf \left\{\frac{\mu_{D_{2}}(\hat{d} f(0)(x))}{\mu_{D_{1}}(x)}: x \in X, \mu_{D_{1}}(x)>0\right\}
$$

$$
\Lambda_{f}=\sup \left\{\frac{\mu_{D_{2}}(\hat{d} f(0)(x))}{\mu_{D_{1}}(x)}: x \in X, \mu_{D_{1}}(x)>0\right\} .
$$

Here $\hat{d} f(0)=\hat{d}^{1} f(0)$ is the linear part of the Taylor series of $f$ at the origin 0 of $X$.

THEOREM 1. Let $X$ and $Y$ be complex normed spaces. Suppose that

(i) $D_{1}$ is a balanced domain in $X$,

(ii) $D_{2}$ is a bounded convex balanced domain in $Y$, Then

(iii) $f: D_{1} \rightarrow D_{2}$ is a holomorphic mapping with $f(0)=0$.

(a) $\mu_{D_{2}}(f(x)) \leqq \mu_{D_{1}}(x) \quad\left(x \in D_{1}\right)$,

(b) $\mu_{D_{2}}(\hat{d} f(0)(x)) \leqq \mu_{D_{1}}(x) \quad\left(x \in D_{1}\right)$,

(c) if $D_{1} \neq X$, then $0 \leqq \tilde{\lambda}_{f} \leqq \tilde{\Lambda}_{f} \leqq 1$.

The equality $\tilde{\lambda}_{f}=1$ holds if and only if the equality $\mu_{D_{2}}(f(x))=\mu_{D_{1}}(x)$ holds for all $x \in D_{1}$.

Proof. We first note that since $D_{2}$ is bounded, convex and balanced, $\mu_{D_{2}}$ is a norm on $Y$ and that since $D_{1}$ is balanced, the power series

$$
\sum_{k=1}^{\infty} \frac{1}{k !} \hat{d}^{k} f(0)(x)
$$


converges to $f$ uniformly on every compact subset of $D_{1}$. (See [1], Corollary 5.2).

Take a point $x_{0} \in D_{1}$ with $\mu_{D_{1}}\left(x_{0}\right)>0$. Put $y=\mu_{D_{1}}\left(x_{0}\right)^{-1} x_{0}$. Then $y \in \partial D_{1}$. Let $\varphi$ be a continuous linear functional on $Y$ of norm 1 , i.e.,

$$
\|\varphi\|=\sup \left\{|\varphi(x)|: x \in Y, \mu_{D_{2}}(x)=1\right\}=1 .
$$

We define the function $g$ by

$$
g(\zeta)=\varphi(f(\zeta y)) \quad(\zeta \in C,|\zeta|<1) .
$$

Then $g$ is holomorphic and $|g(\zeta)| \leqq 1$ in $\Delta=\{\zeta \in C:|\zeta|<1\}$, and $g(0)=0$. Now applying the classical Schwarz lemma we have

$$
\begin{gathered}
|g(\zeta)| \leqq|\zeta| \quad(\zeta \in \Delta), \\
\left|g^{\prime}(0)\right| \leqq 1 .
\end{gathered}
$$

Since $g^{\prime}(0)=\varphi(\hat{d} f(0)(y))=\mu_{D_{1}}\left(x_{0}\right)^{-1} \varphi\left(\hat{d} f(0)\left(x_{0}\right)\right)$, these inequalities imply

$$
\begin{gathered}
\left|\varphi\left(f\left(x_{0}\right)\right)\right| \leqq \mu_{D_{1}}\left(x_{0}\right), \\
\left|\varphi\left(\hat{d} f(0)\left(x_{0}\right)\right)\right| \leqq \mu_{D_{1}}\left(x_{0}\right) .
\end{gathered}
$$

The Hahn-Banach theorem assures the existence of continuous linear functionals $\varphi_{0}$ and $\varphi_{1}$ on $Y$ of norm 1 such that $\varphi_{0}\left(f\left(x_{0}\right)\right)=\mu_{D_{2}}\left(f\left(x_{0}\right)\right)$ and $\varphi_{1}\left(\hat{d} f(0)\left(x_{0}\right)\right)=$ $\mu_{D_{2}}\left(\hat{d} f(0)\left(x_{0}\right)\right)$. Hence we obtain the inequalites

$$
\begin{gathered}
\mu_{D_{2}}\left(f\left(x_{0}\right)\right) \leqq \mu_{D_{1}}\left(x_{0}\right), \\
\mu_{D_{2}}\left(\hat{d} f(0)\left(x_{0}\right)\right) \leqq \mu_{D_{1}}\left(x_{0}\right) .
\end{gathered}
$$

Suppose that $x_{0} \in D_{1}$ and $\mu_{D_{1}}\left(x_{0}\right)=0$. For every $\varepsilon>0$ there is a positive number $t$ such that $0<t<\varepsilon$ and $t^{-1} x_{0} \in D_{1}$. Considering the function

we have

$$
g(\zeta)=\varphi\left(f\left(\zeta t^{-1} x_{0}\right)\right) \quad(\zeta \in \Delta),
$$

$$
\mu_{D_{2}}\left(f\left(x_{0}\right)\right) \leqq t, \quad \mu_{D_{2}}\left(\hat{d} f(0)\left(x_{0}\right)\right) \leqq t .
$$

Hence $\mu_{D_{2}}\left(f\left(x_{0}\right)\right)=\mu_{D_{2}}\left(\hat{d} f(0)\left(x_{0}\right)\right)=0$. Thus (a) and (b) are proved.

Now the inequality

$$
0 \leqq \tilde{\lambda}_{f} \leqq \tilde{\Lambda}_{f} \leqq 1
$$

is an immediate consequence of (b).

Suppose that $\tilde{\lambda}_{f}=1$. Then $\mu_{D_{2}}(\hat{d} f(0)(x))=\mu_{D_{1}}(x)$ for all $x \in X$. Let $x_{0} \in D_{1}$. If $\mu_{D_{1}}\left(x_{0}\right)=0$, then $\mu_{D_{2}}\left(f\left(x_{0}\right)\right)=0$ as we have shown. If $\mu_{D_{1}}\left(x_{0}\right)>0$, we consider the function

$$
g_{1}(\zeta)=\varphi_{1}(f(\zeta y)), \quad y=\mu_{D_{1}}\left(x_{0}\right)^{-1} x_{0}
$$


Then

$$
g_{1}^{\prime}(0)=\mu_{D_{1}}\left(x_{0}\right)^{-1} \varphi_{1}\left(\hat{d} f(0)\left(x_{0}\right)\right)=\mu_{D_{1}}\left(x_{0}\right)^{-1} \mu_{D_{2}}\left(\hat{d} f(0)\left(x_{0}\right)\right)=1
$$

and so $g_{1}(\zeta)=\zeta$ for all $\zeta \in \Delta$. Hence

$$
\mu_{D_{1}}\left(x_{0}\right)=g_{1}\left(\mu_{D_{1}}\left(x_{0}\right)\right)=\varphi_{1}\left(f\left(x_{0}\right)\right) \leqq\left\|\varphi_{1}\right\| \mu_{D_{2}}\left(f\left(x_{0}\right)\right)=\mu_{D_{2}}\left(f\left(x_{0}\right)\right) .
$$

Consequently we obtain that

$$
\mu_{D_{2}}(f(x))=\mu_{D_{1}}(x) \quad\left(x \in D_{1}\right) .
$$

Conversely, suppose that $\mu_{D_{2}}\left(f\left(x_{0}\right)\right)=\mu_{D_{1}}\left(x_{0}\right)$ for some $x_{0} \in D_{1}$ with $\mu_{D_{1}}\left(x_{0}\right)$ $>0$. We consider the function

$$
g_{0}(\zeta)=\varphi_{0}(f(\zeta y)), \quad y=\mu_{D_{1}}\left(x_{0}\right)^{-1} x_{0} .
$$

Since $g_{0}\left(\mu_{D_{1}}\left(x_{0}\right)\right)=\mu_{D_{1}}\left(x_{0}\right)$, we have $g_{0}(\zeta)=\zeta$ for all $\zeta \in \Delta$ and so $g_{0}^{\prime}(0)=1$. Hence we obtain

$$
\mu_{D_{1}}\left(x_{0}\right)=\varphi_{0}\left(\hat{d} f(0)\left(x_{0}\right)\right) \leqq \mu_{D_{2}}\left(\hat{d} f(0)\left(x_{0}\right)\right) .
$$

Thus if $\mu_{D_{2}}(f(x))=\mu_{D_{1}}(x)$ for all $x \in D_{1}$, then $\tilde{\lambda}_{f} \geqq 1$ and hence $\tilde{\lambda}_{f}=1$.

Remarks. If $\mu_{D_{2}}\left(f\left(x_{0}\right)\right)=\mu_{D_{1}}\left(x_{0}\right)$ for some $x_{0} \in D_{1}$ with $\mu_{D_{1}}\left(x_{0}\right)>0$, then $\tilde{\Lambda}_{f}=1$. However, $\tilde{\Lambda}_{f}=1$ does not imply that there exists a point $x_{0} \in D_{1}$ with $\mu_{D_{1}}\left(x_{0}\right)>0$ such that $\mu_{D_{2}}\left(f\left(x_{0}\right)\right)=\mu_{D_{1}}\left(x_{0}\right)$. Indeed, let $X=Y=c_{00}$ and $D_{1}=D_{2}=\left\{x \in c_{00}:\|x\|\right.$ $<1\}$. Here $c_{00}$ is the vector space of all sequences $x=\left(x_{1}, x_{2}, \cdots, x_{n}, \cdots\right)$ of complex numbers having only a finite number of non-vanishing terms, with norm

$$
\|x\|=\max _{n}\left|x_{n}\right| .
$$

The mapping $f: X \rightarrow Y$ defined by

$$
f:\left(x_{1}, x_{2}, \cdots, x_{n}, \cdots\right) \longmapsto\left(\frac{1}{2} x_{1}, \frac{2}{3} x_{2}, \cdots, \frac{n}{n+1} x_{n}, \cdots\right)
$$

maps $D_{1}$ into $D_{2}$ and satisfies $f(0)=0$ and $\tilde{\Lambda}_{f}=1$. But $\mu_{D_{2}}(f(x))=\|f(x)\| \neq\|x\|$ $=\mu_{D_{1}}(x)$ for every $x \in D_{1}$ with $\mu_{D_{1}}(x)=\|x\|>0$.

Moreover, we consider the mapping $g: X \rightarrow Y$ defined by

$$
g:\left(x_{1}, x_{2}, x_{3}, \cdots, x_{n}, \cdots\right) \longmapsto\left(x_{1}^{2}, x_{1}, x_{2}, \cdots, x_{n-1}, \cdots\right) .
$$

Then $g$ maps $D_{1}$ into $D_{2}$ and satisfies $g(0)=0$ and $\tilde{\lambda}_{g}=1$. But $g \neq \hat{d} g(0)$. Thus $\tilde{\lambda}_{g}=1$ does not imply that $g=\hat{d} g(0)$.

COROLlary. Let $X$ and $Y$ be complex normed spaces. If $f: X \rightarrow Y$ is a holomorphic mapping which satisfies $\|f(x)\|=\|x\|$ for all $x \in X$, then $f$ is linear.

Proof. Let $M$ be a positive number. By the assumptions it follows that $f$ 
maps $B(0, M)$ into $B(0, M)$ and satisfies $f(0)=0$ and

$$
\mu_{B(0, M)}(f(x))=\mu_{B(0, M)}(x) \quad(x \in B(0, M)) .
$$

Hence by Theorem 1 we have $\tilde{\lambda}_{f}=1$, and so

$$
\mu_{B(0, M)}(\hat{d} f(0)(x))=\mu_{B(0, M)}(x) \quad(x \in X) .
$$

Therefore it follows that the equality

$$
\|f(x)\|=\|\hat{d} f(0)(x)\|
$$

holds for all $x \in X$. Replacing $x$ by $\zeta x$ we have

$$
\left\|\sum_{k=1}^{\infty} \frac{1}{k !} \zeta^{k-1} \hat{d}^{k} f(0)(x)\right\|=\|\hat{d} f(0)(x)\| \quad(x \in X, \zeta \in \boldsymbol{C}) .
$$

Let $\varphi$ be a continuous linear functional on $Y$. Then the function

$$
h(\zeta)=\varphi\left(\sum_{k=1}^{\infty} \frac{1}{k !} \zeta^{k-1} \hat{d}^{k} f(0)(x)\right)
$$

is holomorphic and bounded in $\boldsymbol{C}$. Hence the Liouville theorem says that $h$ is constant. Since the dual space $Y^{*}$ of $Y$ separates points on $Y$, it now follows that

$$
\sum_{k=2}^{\infty} \frac{1}{k !} \zeta^{k-1} \hat{d}^{k} f(0)(x)=0 \quad(\zeta \in C) .
$$

Therefore we conclude that $f=\hat{d} f(0)$.

Next we prove a theorem which gives a lower bound for $\mu_{D_{\mathbf{2}}}(f(x)), x \in D_{1}$. In our proof the following fact plays an important role.

Proposition. Let $X$ be a complex normed space and $D$ be a convex balanced domain in $X$. Let $k_{D}$ denote the Kobayashi pseudodistance of $D$. Then

$$
k_{D}(0, x)=\frac{1}{2} \log \frac{1+\mu_{D}(x)}{1-\mu_{D}(x)}
$$

and

$$
\left\{x \in D: k_{D}(0, x)<\alpha\right\}=r D, \quad r=\frac{e^{2 \alpha}-1}{e^{2 \alpha}+1} .
$$

(See [2], Theorem IV.1.8).

THEOREM 2. Let $X$ and $Y$ be complex normed spaces. Suppose that

(i) $D_{1}$ is a balanced domain in $X$,

(ii) $D_{2}$ is a bounded convex balanced domain in $Y$, Then

(iii) $f: D_{1} \rightarrow D_{2}$ is a holomorphic mapping with $f(0)=0$. 


$$
\mu_{D_{2}}(f(x)) \geqq \frac{\mu_{D_{1}}(x)\left(\tilde{\lambda}_{f}-\mu_{D_{1}}(x)\right)}{1-\tilde{\lambda}_{f} \mu_{D_{1}}(x)} \quad\left(x \in D_{1}\right) .
$$

Proof. Take a point $x_{0} \in D_{1}$ with $\mu_{D_{1}}\left(x_{0}\right)>0$. Put $y=\mu_{D_{1}}\left(x_{0}\right)^{-1} x_{0}$ and define the mapping $g$ from $\Delta$ into $D_{2}$ by

$$
g(\zeta)=f(\zeta y) \quad(\zeta \in \Delta) .
$$

Since $D_{1}$ is balanced, the Taylor series of $f$ at 0

$$
\sum_{k=1}^{\infty} \frac{1}{k !} \hat{d}^{k} f(0)(x)
$$

converges to $f$ uniformly on every compact subset of $D_{1}$. Hence we have

$$
g(\zeta)=\sum_{k=1}^{\infty} \frac{1}{k !} \zeta^{k} \hat{d}^{k} f(0)(y) \quad(\zeta \in \Delta),
$$

and hence we can write

$$
g(\zeta)=\zeta h(\zeta) \quad(\zeta \in \Delta),
$$

where $h$ is a holomorphic mapping from $\Delta$ into $Y$ with the Taylor series

$$
h(\zeta)=\sum_{k=1}^{\infty} \frac{1}{k !} \zeta^{k-1} \hat{d}^{k} f(0)(y)
$$

at 0 . Let $0<t<1$. By Proposition and the definition of $k_{D_{2}}$ we have that if $\zeta h(\zeta) \neq 0$, then

$$
k_{D_{2}}(0, t \zeta h(\zeta))<k_{D_{2}}(0, \zeta h(\zeta))=k_{D_{2}}(g(0), g(\zeta)) \leqq \frac{1}{2} \log \frac{1+|\zeta|}{1-|\zeta|},
$$

and so

$$
t \zeta h(\zeta) \in|\zeta| D_{2}
$$

Since $D_{2}$ is balanced, this implies that

$$
\operatorname{th}(\zeta) \in D_{2} \quad(\zeta \in \Delta, \zeta \neq 0) .
$$

By Theorem 1, $h(0)=\hat{d} f(0)(y) \in \bar{D}_{2}$ and so $t h(0) \in D_{2}$. Thus $t h$ is also a holomorphic mapping from $\Delta$ into $D_{2}$. Therefore by Proposition and the definition of $k_{D_{2}}$ we have the following two inequalities:

$$
\begin{aligned}
k_{D_{2}}(0, t h(0))=k_{D_{2}}(0, t \hat{d} f(0)(y)) & =\frac{1}{2} \log \frac{1+t \mu_{D_{2}}(\hat{d} f(0)(y))}{1-t \mu_{D_{2}}(\hat{d} f(0)(y))} \\
& \geqq \frac{1}{2} \log \frac{1+t \tilde{\lambda}_{f}}{1-t \tilde{\lambda}_{f}},
\end{aligned}
$$

and

$$
k_{D_{2}}(\operatorname{th}(0), \operatorname{th}(\zeta)) \leqq \frac{1}{2} \log \frac{1+|\zeta|}{1-|\zeta|}
$$


Moreover, by the triangle inequality we have

$$
k_{D_{2}}(0, \operatorname{th}(\zeta)) \geqq k_{D_{2}}(0, \operatorname{th}(0))-k_{D_{2}}(t h(0), \operatorname{th}(\zeta)) .
$$

Combining these inequalities we obtain

$$
k_{D_{2}}(0, \operatorname{th}(\zeta)) \geqq \frac{1}{2} \log \frac{\left(1+t \tilde{\lambda}_{f}\right)(1-|\zeta|)}{\left(1-t \tilde{\lambda}_{f}\right)(1+|\zeta|)} .
$$

Now this inequality and Proposition show that

$$
\begin{aligned}
\mu_{D_{2}}(\operatorname{tg}(\zeta)) & =|\zeta| \mu_{D_{2}}(\operatorname{th}(\zeta))=|\zeta| \Phi\left(k_{D_{2}}(0, \operatorname{th}(\zeta))\right) \\
& \geqq|\zeta| \Phi\left(\frac{1}{2} \log \frac{\left(1+t \tilde{\lambda}_{f}\right)(1-|\zeta|)}{\left(1-t \tilde{\lambda}_{f}\right)(1+|\zeta|)}\right)=\frac{|\zeta|\left(t \tilde{\lambda}_{f}-|\zeta|\right)}{1-t \tilde{\lambda}_{f}|\zeta|},
\end{aligned}
$$

where $\Phi(s)=\left(e^{2 s}-1\right) /\left(e^{2 s}+1\right)$. Letting $t \rightarrow 1$ and putting $\zeta=\mu_{D_{1}}\left(x_{0}\right)$ we obtain the desired inequality

$$
\mu_{D_{2}}\left(f\left(x_{0}\right)\right) \geqq \frac{\mu_{D_{1}}\left(x_{0}\right)\left(\tilde{\lambda}_{f}-\mu_{D_{1}}\left(x_{0}\right)\right)}{1-\tilde{\lambda}_{f} \mu_{D_{1}}\left(x_{0}\right)}
$$

4. Finally we study the limits of sequences of automorphisms of bounded domains. If $D$ is a bounded domain in $C^{n}$ and if $f: D \rightarrow D$ is a pointwise limit of a sequence $\left\{F_{n}\right\}$ of automorphisms of $D$, then $f$ is also an automorphism of $D$. This follows from the fact that $\left\{F_{n}\right\}$ has a subsequence $\left\{F_{n_{k}}\right\}$ which converges to $f$ uniformly on every compact subset of $D$. (See [7], pp. 78-82). However, in the case that $D$ is a bounded domain in a complex normed space $X$ the limit $f: D \rightarrow D$ of a sequence of automorphisms of $D$ need not be an automorphism of $D$. In this section using Theorems 1 and 2 we prove that $f$ is one-to-one.

Let $X$ be a complex normed space and $D$ be a domain in $X$. The automorphisms of $D$ are the biholomorphic mappings from $D$ onto $D$. We denote by $\operatorname{Aut}(D)$ the group of all automorphisms of $D$. We begin with a simple example.

Example. Let $X=c_{00}$ and $D=\{x \in X:\|x\|<1\}$. Define the mappings $F_{n}$, $n=1,2, \cdots$, and $f$ by

and

$$
F_{n}:\left(x_{1}, x_{2}, x_{3}, \cdots, x_{n}, x_{n+1}, \cdots\right) \longmapsto\left(x_{n}, x_{1}, x_{2}, \cdots, x_{n-1}, x_{n+1}, \cdots\right)
$$

$$
f:\left(x_{1}, x_{2}, x_{3}, \cdots, x_{n}, \cdots\right) \longmapsto\left(0, x_{1}, x_{2}, \cdots, x_{n-1}, \cdots\right) .
$$

Then $D$ is a bounded domain in $X$ and $F_{n} \in \operatorname{Aut}(D), n=1,2, \cdots$. Moreover,

$$
f(x)=\lim _{n \rightarrow \infty} F_{n}(x) \quad(x \in D) .
$$

However, $f \notin \operatorname{Aut}(D)$. 

define

We next prove two lemmas. For a holomorphic mapping $f: D \rightarrow X$ we

and

$$
\lambda_{f}(a)=\inf \left\{\frac{\|\hat{d} f(a)(x)\|}{\|x\|}: x \in X, x \neq 0\right\} \quad(a \in D),
$$

$$
\Lambda_{f}(a)=\sup \left\{\frac{\|\hat{d} f(a)(x)\|}{\|x\|}: x \in X, x \neq 0\right\} \quad(a \in D) .
$$

Here we note that if $f$ maps $B\left(0, r_{1}\right)$ into $B\left(0, r_{2}\right)$, then

$$
\lambda_{f}(0)=\frac{r_{2}}{r_{1}} \tilde{\lambda}_{f}, \quad \Lambda_{f}(0)=\frac{r_{2}}{r_{1}} \tilde{\Lambda}_{f} .
$$

LEMMA 1. Let $D$ be a bounded domain in $X$. Let $F \in \operatorname{Aut}(D)$ and $a \in D$. If $M, r$ and $\rho$ are positive numbers such that $D \subset B(0, M), B(a, r) \subset D$ and $B(F(a), \rho) \subset D$, then

$$
\frac{\rho}{2 M} \leqq \lambda_{F}(a) \leqq \Lambda_{F}(a) \leqq \frac{2 M}{r} .
$$

Proof. Since $F^{-1} \circ F=F_{\circ} F^{-1}=$ id., we have $\hat{d} F^{-1}(F(a)) \circ \hat{d} F(a)=\hat{d} F(a) \circ$ $\hat{d} F^{-1}(F(a))=$ id. Hence it follows that $\hat{d} F(a) \in \operatorname{Aut}(X)$ and $\lambda_{F}(a) \Lambda_{F-1}(F(a))=1$.

Put $G(x)=F(x+a)-F(a)$. Then $G$ maps $B(0, r)$ into $B(0,2 M)$ and $G(0)$ $=0$. Hence, by Theorem 1, we obtain

$$
\Lambda_{F}(a)=\Lambda_{G}(0)=\frac{2 M}{r} \tilde{\Lambda}_{G} \leqq \frac{2 M}{r} .
$$

On the other hand, since $F^{-1}$ maps $B(F(a), \rho)$ into $B(0, M)$, we have

$$
\lambda_{F}(a)=\frac{1}{\Lambda_{F^{-1}}(F(a))} \geqq \frac{\rho}{2 M} .
$$

Lemma 2. Let $D$ be a bounded domain in $X$. Let $F \in$ Aut $(D), a \in D$ and $\lambda_{F}(a) \geqq \lambda_{0}>0$. Let $M$ and $r$ be positive numbers such that $B(a, r) \subset D \subset B(0, M)$. If $0<t<\left(r^{2} \lambda_{0} / 4 M\right)$, then

$$
F(B(a, t)) \supset B\left(F(a), \frac{\lambda_{0}}{2} t\right) .
$$

Proof. Put $G(x)=F(x+a)-F(a)$. Then $G$ maps $B(0, r)$ into $B(0,2 M)$ and $G(0)=0$. Hence applying Theorem 2 to $G$ we have

$$
\|G(x)\| \geqq \frac{2 M}{r^{2}} \frac{\|x\|\left(r^{2} \lambda_{G}(0)-2 M\|x\|\right)}{2 M-\lambda_{G}(0)\|x\|} \quad(x \in B(0, r)) .
$$

Since $\lambda_{G}(0)=\lambda_{F}(a) \geqq \lambda_{0}$, we have

$$
\|F(x)-F(a)\| \geqq \frac{2 M}{r^{2}} \frac{\|x-a\|\left(r^{2} \lambda_{0}-2 M\|x-a\|\right)}{2 M-\lambda_{0}\|x-a\|} \quad(x \in B(a, r)) .
$$


Hence using the inequality $\lambda_{0} \leqq \lambda_{F}(a) \leqq \Lambda_{F}(a) \leqq(2 M / r)$, we obtain that if $\|x-a\|$ $\leqq\left(r^{2} \lambda_{0} / 4 M\right)$, then

$$
\|F(x)-F(a)\| \geqq \frac{4 M^{2} \lambda_{0}}{8 M^{2}-r^{2} \lambda_{0}^{2}}\|x-a\| \geqq \frac{\lambda_{0}}{2}\|x-a\| .
$$

Since $F \in \operatorname{Aut}(D)$, this inequality shows that if $0<t<\left(r^{2} \lambda_{0} / 4 M\right)$, then

$$
F(B(a, t)) \supset B\left(F(a), \frac{\lambda_{0}}{2} t\right) .
$$

Now we can prove the following theorem.

Theorem 3. Let $D$ be a bounded domain in $X$. Suppose that $f: D \rightarrow D$ is a pointwise limit of a sequence $\left\{F_{n}\right\}$ of automorphisms of $D$. Then $f$ is one-toone in $D$.

Proof. Assume that there exist two distinct points $a_{1}$ and $a_{2}$ in $D$ such that $f\left(a_{1}\right)=f\left(a_{2}\right)=b$. Since $b \in D$, there is a positive number $\rho$ with $B(b, 2 \rho)$ $\subset D$. Hence we can choose a positive integer $n_{0}$ such that if $n>n_{0}$, then $B\left(F_{n}\left(a_{\imath}\right), \rho\right) \subset D, i=1,2$. Take positive numbers $M, r_{1}$ and $r_{2}$ such that $B(0, M)$ $\supset D, B\left(a_{1}, r_{1}\right) \subset D$ and $B\left(a_{2}, r_{2}\right) \subset D$. Then, by Lemma 1 , we have, if $n>n_{0}$, then

$$
\lambda_{F_{n}}\left(a_{\imath}\right) \geqq \frac{\rho}{2 M} \quad(i=1,2),
$$

and hence, by Lemma 2 , if $n>n_{0}$ and $0<t<\left(r_{i}^{2} \rho / 8 M^{2}\right)$, then

$$
F_{n}\left(B\left(a_{\imath}, t\right)\right) \supset B\left(F_{n}\left(a_{\imath}\right), \frac{\rho}{4 M} t\right) \quad(i=1,2) .
$$

On the other hand, we can choose a positive number $t$ and a positive integer $n$ satisfying conditions:

(i) $0<t<\min \left\{\frac{r_{1}^{2} \rho}{8 M^{2}}, \frac{r_{2}^{2} \rho}{8 M^{2}}\right\}$, and $B\left(a_{1}, t\right) \cap B\left(a_{2}, t\right)=\emptyset$,

(ii) $n>n_{0}$ and $B\left(F_{n}\left(a_{1}\right), \frac{\rho}{4 M} t\right) \cap B\left(F_{n}\left(a_{2}\right), \frac{\rho}{4 M} t\right) \neq \emptyset$.

These facts contradict that $F_{n} \in$ Aut $(D)$. Therefore it follows that $f$ is one-toone in $D$.

\section{REFERENCES}

[1] J.A. BARroso, Introduction to Holomorphy, North-Holland Math. Studies, 106, Amsterdam, 1985.

[2] T. Franzoni and E. Vesentini, Holomorphic Maps and Invariant Distances. North-Holland Math. Studies, 40, Amsterdam, 1980. 
[3] S. J. Greenfield and N.R. Wallach, Automorphism groups of bounded domains in Banach spaces, Trans. Amer. Math. Soc., 166 (1972), 45-57.

[4] L. A. HARris, Schwarz's lemma in normed linear spaces, Proc. Nat. Acad. Sc1. U.S.A., 62 (1969), 1014-1017.

[5] L.A. HARRIS, A continuous form of Schwarz's lemma in normed linear spaces, Pacific J. Math., 38 (1971), 635-639.

[6] L. NAchBin, Topology on Spaces of Holomorphic Mappings, Ergebnisse der Mathematik und ihrer Grenzgebiete, 47, Springer-Verlag, Berlin, 1969.

[7] R. Narasimhan, Several Complex Variables, University of Chicago Press, Chicago, 1971.

[8] W. Rudin, Function Theory in the Unit Ball of $C^{n}$, Springer-Verlag, BerlinHeidelberg-New York, 1980.

Department of Mathematics

TOKYo GaKugEI UNIVERSITY

KOGANEI-SHI, TOKYO 184, JAPAN 